\title{
A new simplified design method for steel structures under impulsive loading
}

\author{
C. Colomer Segura \& M. Feldmann \\ Institute of Steel and Lightweight Construction, \\ RWTH Aachen University, Germany
}

\begin{abstract}
The dynamic response of structures under impulsive loading is a complex process where both geometric and material nonlinearities play a very important role. The use of Finite Element calculations is advisable for the design of structures that must withstand impulsive action (blast or impact), but designers very often lack the resources to perform these calculations, thus simplified design methods are often used, mainly based on the reduction of the original system to dynamically equivalent SDOF system.

Most of the existing approaches suffer from shortcomings when having to deal with arbitrary support conditions, to consider catenary effects or to consider interaction effects with surrounding parts of the structure.

In order to overcome these limitations, the Institute of Steel Construction has proposed a new 2-DOF dynamically equivalent system reduction, which allows for the explicit consideration of catenary action including connector failure and opens new possibilities for integration with more complex Finite Element models. Within this new approach, the connectors are treated distinctly, so that a separate assessment of its failure is possible.
\end{abstract}

Keywords: equivalent system, SDOF, system reduction, geometrical nonlinearities, dynamically equivalent system, beam reduction, FEM.

\section{Introduction}

Accidental actions such as explosions can load structures up to their ultimate resistance levels. Especially the members directly affected by the action undergo large plastic deformations, which lead locally to member failure and may affect 
the overall stability of the structure. An accurate prediction of the dynamic response is decisive for a safe and robust design of the structure.

Due to the large amount of physical and geometrical nonlinearities, as well as the highly time dependent behaviour present in explosive events, the modelling and calculation of this processes is subjected to high computational requirements. The most exact and reliable method of predicting the structural response consists of the use of Finite Element Methods (FEM) with explicit solvers. Hereby the calculation of a structure undergoing a given explosive load requires considerable modelling expertise as well as very large computational power, with calculations taking several days to compute. Normally common software packages used for the design of steel structures do not allow for this type of modelling and solving strategies, since they involve a solution in the time domain under consideration of geometrical, material and contact nonlinearities.

On the other hand, simplified design methods offer an affordable, much less time-consuming alternative for predicting the effects of blast action, which can be used by a wide spectrum of structural engineers, without advanced knowledge of the FEM.

The analytical and numerical background for the modelling and calculation of structures under the effect of explosive loads by means of simplified methods was established at the end of the 50s. Motivated by strategic military questions, the US Army performed a very extensive program of experimental, numerical and analytical investigations on this topic. A series of Technical and Engineering Manuals under the title "Design of Structures to Resist the Effects of Atomic Weapons" [1] were published between 1957 and 1961 under the lead of scientists from Massachusetts Institute of Technology (MIT) (e.g. Norris et al. [2]). Especially the working group of Biggs [3] settled the fundamentals for dynamic system reduction, that are still used in present design recommendations [4-7].

In this paper, a brief overview of these two design methodologies, specially focusing on their basic assumptions and limitations, will be given in Section 2. In the scope of this work, the focus lies on the development of a new design methodology for steel structures where one-way components (beam columns) must withstand the explosion. Therefore, the fundamentals of dynamic system reduction focusing on beam elements are presented in Section 3. In order to overcome some known limitations dealing with the effects of geometrical nonlinearities, a new reduction methodology is proposed for including the effects of axial forces and/or boundary conditions in an explicit manner. In Section 4 a validation example of the new methodology is given.

\section{Design methodologies}

The solution of structures under impact loading can be obtained by two different methodologies. These differ very strongly in the amount of modelling detail, calculation time and solution type, so that it is very important to analyse the strengths and weaknesses of each method in order to use them properly. 


\subsection{Finite Element calculations}

The Finite Element Method offers the most exact and realistic description of the problem. By means of beams, shells or volume elements using appropriate material models and contact definition, any type of problem can be addressed. With increasing level of detail, the amount of system degrees of freedom increases strongly.

Due to the short duration of the load application, the system is excited in very high frequencies. Thus, the use of explicit solvers is strongly recommended for this type of calculations.

The direct simulation of impulsive loaded structures is very demanding in terms of computing time, numeric stability and modelling accuracy. The required small time stepping (due to the very short impulse duration) for the dynamic calculation leads often to numerical convergence problems as well as to very large computing times even for the simulation of few milliseconds of the structural response.

The advantages of the use of the FEM are: (a) the possibility to include details of interest (i.e. bolts) and obtain a direct output of the magnitudes of interest; (b) the solution is in the time domain, hence it is possible to follow the development of the failure mechanism; (c) advanced material models and exact geometrical theories can be considered; (d) interaction effects between different members are considered.

On the other hand, the use of FEM has some disadvantages: (a) the problem setup is quite time consuming and requires advanced expertise; (b) very high time requirements for calculation; and (c) parametric studies are generally not possible.

Especially on early design stages, the use of FEM is not advisable, since the structural design and detailing is still to be determined by iterative calculations.

\subsection{Simplified models}

From a designer point of view, the knowledge of the structural response of a certain given system is of small interest compared to the ability of predicting the behaviour of the same system under variation of different parameters (i.e. connector stiffness, mass distribution, chosen cross-section ...). This knowledge can only be achieved through parametric studies.

The performance of parametric studies for this type of phenomena is, even with today's computer power, not feasible for detailed Finite Element systems (this means including connectors, beam and shell elements), so that simplifications of the system behaviour are assumed in order to allow for this type of studies.

Through the definition of energetic equivalent systems (also called dynamic equivalent systems), the problem complexity can be dramatically reduced, and thus the solution time.

An energetic equivalent system reduces the investigated structure to a single degree of freedom (SDOF) system, which shows the same deformational and 
energetic behaviour than the original system (see fig. 1). The effects of structural damping are neglected here.

Once the reduced model is defined, the motion differential equation of an undamped nonlinear SDOF

$$
M_{E} \cdot \ddot{x}+R_{E}(x)=f_{E}(t)
$$

with $\quad M_{E} \quad$ equivalent mass of the system,

$x, \ddot{x} \quad$ displacement and acceleration of the equivalent mass,

$R_{E}(x)$ equivalent force-displacement relation,

$f_{E}(t)$ equivalent explosive load,

can be solved by simple numerical implementation (see $[3,6,9])$.
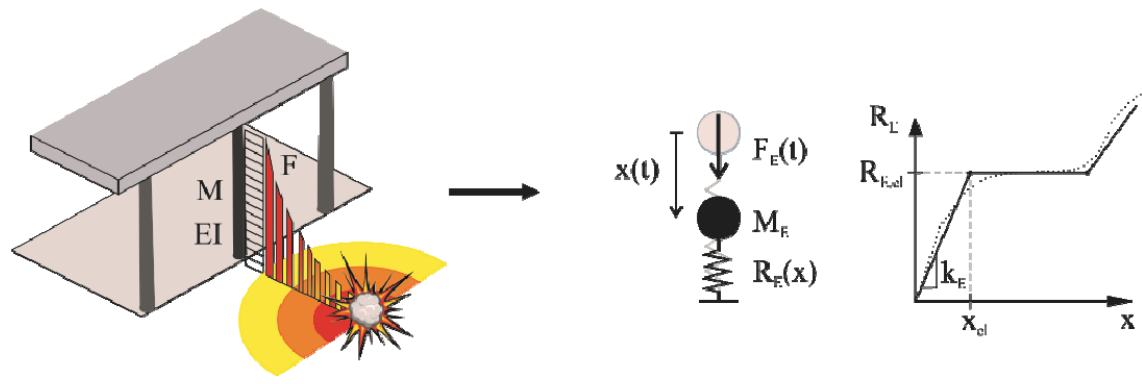

Figure 1: Member reduction to a SDOF with nonlinear force-displacement relation.

In this model, all structural nonlinearities are considered by a nonlinear definition of the force-displacement relation $R_{E}(x)$ of the equivalent spring. While the first approaches from Norris et al. [2] and Biggs [3], considered an elastic-perfectly plastic model, it was very soon observed, that this approach was too conservative, because it neglected the stiffening effect of membrane force activation. Different additions to the $R_{E}(x)$ curve have been proposed in modern codes $[4,5,7]$, being the approach from the Fire and Blast Information Group [6] the most detailed one, proposing a 6-piece curve for $R_{E}(x)$.

For some of these model simplifications, parametric studies have been made under consideration of varying loading $\left(F_{\max }\right.$ peak pressure and $t_{d}$ impulse duration) and varying system properties ( $T$ natural period, $R_{E, e l}$ plastic resistance, $k_{E}$ initial stiffness). For a given set of boundary and loading conditions, the required ductility $\mu=x_{\max } / x_{e l}$ can then be evaluated from diagrams (e.g. fig. 2).

The main advantages of the use of simplified models for dynamic calculations are: (a) its easiness of use and programmability, (b) the adequacy for use in parametric studies and (c) the existence of associated diagrams to quickly assess the required member ductility.

On the other hand, (a) the use of simplified models is limited to the evaluation of a single member (no interaction effects between substructure and main structure); (b) its use is only valid for a given set of boundary conditions; and 
(c) the treatment of geometrical nonlinearities in some approaches is non-trivial and requires a good theoretical background.

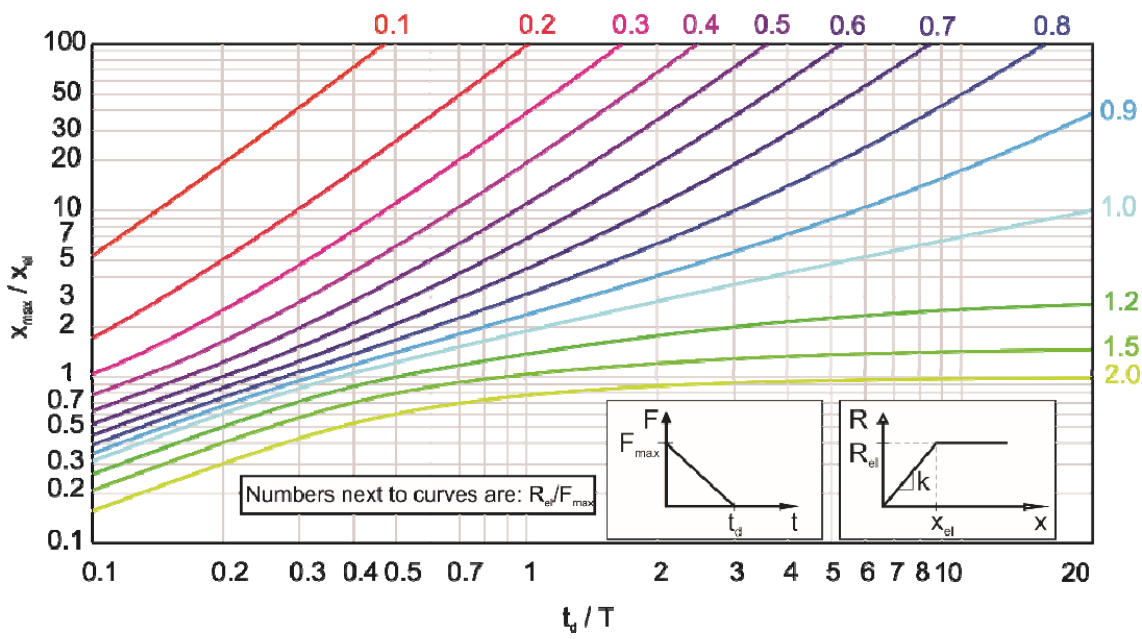

Figure 2: Required member ductility in dependence of the normalised impulse duration $\left(\mathrm{t}_{\mathrm{d}} / \mathrm{T}\right)$ and normalized peak load $\left(\mathrm{R}_{\mathrm{el}} / \mathrm{F}_{\mathrm{max}}\right)$, for an elastic-perfectly plastic system, from [7].

\section{Definition of a new dynamic equivalent system}

Here a new dynamic equivalent system for a generally supported beam element will be presented. Differing from classical approaches mentioned in section 2.2, the new reduced system consists of a mass with 2 DOF (in vertical and horizontal direction) supported by a set of 4 springs as represented in fig. 3 :
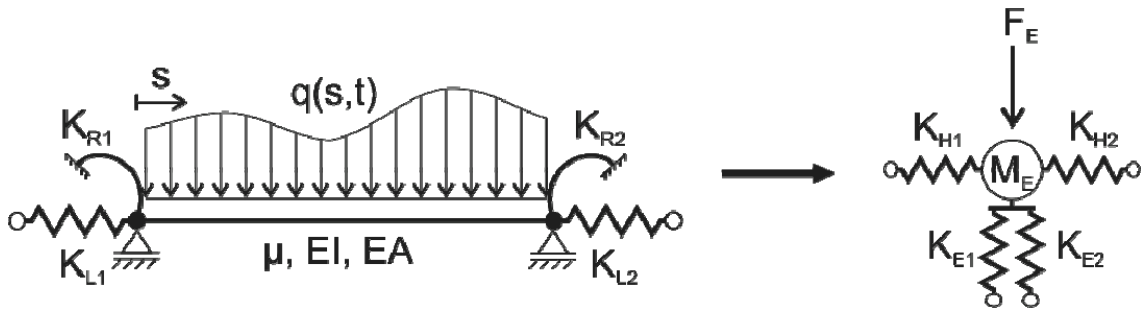

Figure 3: Proposed member reduction to a new dynamic equivalent system.

This system decomposition separates the effects of the beam nonlinearities in two components, i.e. material and geometrical. In this manner, the resistance behaviour due to the flexure of the member (including material nonlinearities 
caused by the yielding of the member or any of the rotational springs $K_{R 1}$ and $\mathrm{K}_{\mathrm{R} 2}$ ) is considered by the two vertical springs $\mathrm{K}_{\mathrm{E} 1}$ and $\mathrm{K}_{\mathrm{E} 2}$.

On the other hand, the geometrical (membrane) nonlinear behaviour is modelled by the two horizontal geometrical nonlinear springs $\mathrm{K}_{\mathrm{H} 1}$ and $\mathrm{K}_{\mathrm{H} 2}$. By the use of a nonlinear material definition, they account as well for connector failure (or axial yielding of the beam).

According to [1], in the energetic analysis of a dynamically excited structure, there are three quantities to be considered: 1) work done or external work, 2) strain energy or internal energy and 3) kinetic energy. The definition of a dynamically equivalent system involves the principle of dynamic similarity, which is merely the requirement that the three mentioned energy components remain equal with those of the given structure.

Assuming a beam element undergoing an arbitrary deformation $\phi(s, t)$ (as in fig. 4), the new equivalent system must be defined by requiring that 1) the deformation behavior, 2) the kinetic energy stored in the lumped mass $\mathrm{M}_{\mathrm{E}}, 3$ ) the strain energy stored in the springs and 4) the external work done by the force $\mathrm{F}_{\mathrm{E}}$ remain equal for both the original and the equivalent system.

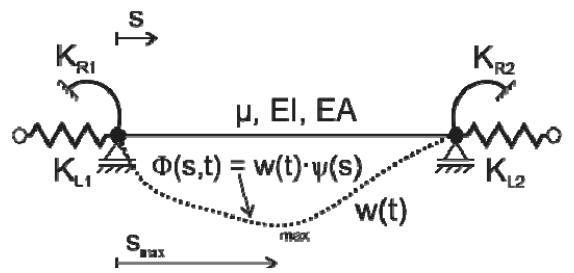

Figure 4: Arbitrary deformation.

\subsection{Equivalent mass}

The equivalent mass of a beam undergoing a deformation $\Phi(\mathrm{s}, \mathrm{t})=\mathrm{w}(\mathrm{t}) \cdot \psi(\mathrm{s})$ (as in fig. 4) is defined as the mass needed in order to absorb the same amount of kinetic energy, assuming that the equivalent mass deforms with the same peak deformation over time $\mathrm{w}(\mathrm{t})$.

$$
E_{v, \mathrm{~S}}=E_{v, \mathrm{E}}
$$

with $\quad E_{v, \mathrm{E}}=\frac{1}{2} \cdot M_{E} \cdot\left(\frac{d w(t)}{d t}\right)^{2}$

$$
\begin{aligned}
& E_{v, \mathrm{~S}}=\frac{1}{2} \cdot\left[\int \mu \cdot \psi(s)^{2} \cdot d s+\sum\left(\mathrm{M}_{\mathrm{i}} \cdot \psi\left(s_{i}, t\right)^{2}\right)\right] \cdot\left(\frac{d w(t)}{d t}\right)^{2} \\
& \text { with } \mu \quad \text { cross-sectional mass (per unit length) } \\
& \mathrm{M}_{\mathrm{i}} \quad \text { concentrated masses at points } \mathrm{s}_{\mathrm{i}}
\end{aligned}
$$

From Eqn. (2), it can be concluded that:

$$
M_{E}=\int \mu \cdot \psi(s)^{2} \cdot d s+\sum \mathrm{M}_{\mathrm{i}} \cdot \psi\left(s_{i}, t\right)^{2}
$$




\subsection{Equivalent load}

The equivalent load acting on a beam undergoing the same deformation $\Phi(\mathrm{s}, \mathrm{t})$ is defined as the concentrated load acting on the equivalent mass that is needed to input the same amount of external work in the system, assuming that the equivalent mass deforms with the same peak deformation over time $\mathrm{w}(\mathrm{t})$.

$$
\Delta E_{S}=\Delta E_{E}
$$

with $\quad \Delta E_{E}=\frac{1}{2} \cdot F_{E}(\mathrm{t}) \cdot \mathrm{w}(\mathrm{t})$

$$
\begin{aligned}
& \Delta E_{S}=\frac{1}{2} \cdot\left[\int \mathrm{q}(\mathrm{s}, \mathrm{t}) \cdot \psi(s) \cdot d s+\sum\left(\mathrm{P}_{\mathrm{i}} \cdot \psi\left(s_{i}, t\right)\right)\right] \cdot \mathrm{w}(\mathrm{t}) \\
& \text { with } \quad \begin{array}{cl}
\mathrm{q}(\mathrm{s}, \mathrm{t}) & \text { distributed load (per unit length) } \\
\mathrm{P}_{\mathrm{i}} & \text { concentrated loads at points } \mathrm{s}_{\mathrm{i}}
\end{array}
\end{aligned}
$$

From Eqn. (4), it can be concluded that:

$$
F_{E}(\mathrm{t})=\int \mathrm{q}(\mathrm{s}, \mathrm{t}) \cdot \psi(s) \cdot d s+\sum\left(\mathrm{P}_{\mathrm{i}} \cdot \psi\left(s_{i}, t\right)\right)
$$

\subsection{Equivalent stiffness for vertical springs $K_{E 1}$ and $K_{E 2}$}

The equivalent stiffness for the vertical springs must absorb the same amount of bending strain energy as the original beam undergoing a deformation $\Phi(s, t)$. This definition is the same as in the classical Biggs' approach [3]. The bending strain energy equilibrium can be formulated as:

$$
E_{\kappa, \mathrm{S}}=E_{\kappa, \mathrm{E}}
$$

with $\quad E_{\kappa, \mathrm{E}}=\frac{1}{2} \cdot k_{E} \cdot w^{2}$

$$
\begin{aligned}
& E_{\kappa, \mathrm{S}}=\left(\int E I \cdot\left(\frac{\mathrm{d}^{2} \psi(s)}{\mathrm{ds}^{2}}\right)^{2} \cdot d \mathrm{~s}+k_{R 1} \cdot\left(\frac{d \psi(0)}{d s}\right)^{2}+k_{R 2} \cdot\left(\frac{d \psi(L)}{d s}\right)^{2}\right) \cdot \frac{w^{2}}{2} \\
& \text { with EI bending stiffness of the beam } \\
& \mathrm{k}_{\mathrm{R} 1}, \mathrm{k}_{\mathrm{R} 2} \text { rotational stiffness of springs } \mathrm{K}_{\mathrm{R} 1} \text { and } \mathrm{K}_{\mathrm{R} 2}
\end{aligned}
$$

From Eqn. (6), it can be concluded that:

$$
k_{E}=\int E I \cdot\left(\frac{d^{2} \psi(s)}{d s^{2}}\right)^{2} \cdot d s+k_{1} \cdot\left(\frac{d \psi(0)}{d s}\right)^{2}+k_{2} \cdot\left(\frac{d \psi(L)}{d s}\right)^{2}
$$

The stiffness $\mathrm{k}_{\mathrm{E}}$ of the vertical springs in the equivalent model is distributed according to the following weighting function:

$$
k_{E 1}=k_{E} \cdot \frac{5 \cdot \mathrm{k}_{1}+3 \cdot \mathrm{k}_{2}+\mathrm{k}_{1} \cdot \mathrm{k}_{2}+12}{4 \cdot \mathrm{k}_{1}+4 \cdot \mathrm{k}_{2}+\mathrm{k}_{1} \cdot \mathrm{k}_{2}+12} \text { and } k_{E 2}=k_{E} \cdot \frac{3 \cdot \mathrm{k}_{1}+5 \cdot \mathrm{k}_{2}+\mathrm{k}_{1} \cdot \mathrm{k}_{2}+12}{4 \cdot \mathrm{k}_{1}+4 \cdot \mathrm{k}_{2}+\mathrm{k}_{1} \cdot \mathrm{k}_{2}+12}
$$

which corresponds to the elastic distribution coefficients for a two-way supported beam with rotational stiffness $\mathrm{k}_{\mathrm{R} 1}=\mathrm{k}_{1} \cdot \mathrm{EI} / \mathrm{L}$ and $\mathrm{k}_{\mathrm{R} 2}=\mathrm{k}_{2} \cdot \mathrm{EI} / \mathrm{L}$. In a perfectly plastic developed state $\mathrm{k}_{\mathrm{E} 1}=\mathrm{k}_{\mathrm{E} 2}$. 


\subsection{Equivalent stiffness for horizontal springs $K_{\mathrm{H} 1}$ and $K_{\mathrm{H} 2}$}

The horizontal springs $\mathrm{K}_{\mathrm{H} 1}$ and $\mathrm{K}_{\mathrm{H} 2}$ must absorb the same amount of membrane strain energy as the original beam undergoing a deformation $\Phi(\mathrm{s}, \mathrm{t})$. In order to correctly evaluate the cumulated membrane (axial) strain energy, an additional deformation hypothesis $\mathrm{u}(\mathrm{x})$ as in fig. 5 needs to be defined.
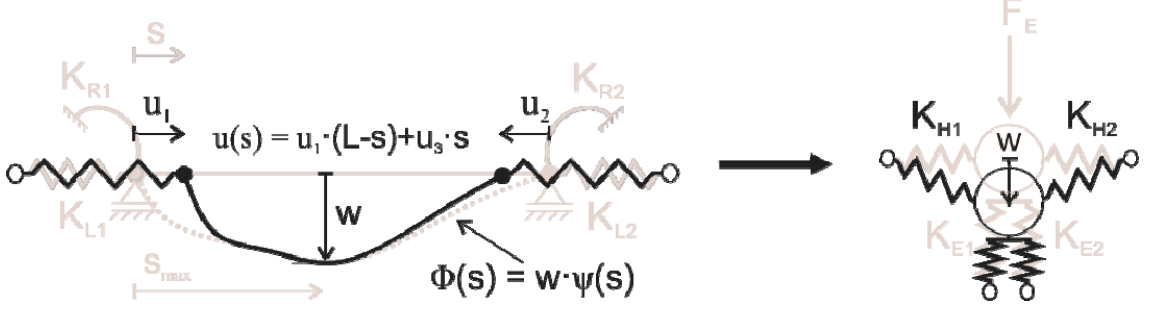

Figure 5: Deformation hypothesis in axial direction and equivalent model for the absorption of membrane strain energy.

In order to calculate the equivalent spring stiffnesses $\mathrm{k}_{\mathrm{H} 1}$ and $\mathrm{k}_{\mathrm{H} 2}$ of the springs $\mathrm{K}_{\mathrm{H} 1}$ and $\mathrm{K}_{\mathrm{H} 2}$, the use of a nonlinear strain definition is needed. Assuming:

$$
\varepsilon(s)=\frac{d u(s)}{d s}+\frac{1}{2} \cdot\left(\frac{d \Phi(s)}{d s}\right)^{2}
$$

the membrane strain energy accumulated in the original system from fig. 5 can be calculated with eqn. (10).

$$
\begin{gathered}
E_{\varepsilon, S}=\frac{k_{L 1}}{2} \cdot u_{1}^{2}+\frac{k_{L 2}}{2} \cdot u_{2}^{2}+\frac{E A}{2} \cdot \int_{0}^{L} \varepsilon(s)^{2} \cdot d s=\frac{k_{L 1}}{2} \cdot u_{1}^{2}+\frac{k_{L 2}}{2} \cdot u_{3}^{2}+\frac{E A}{2} \cdot \frac{\left(u_{1}-u_{2}\right)^{2}}{L}+ \\
\frac{E A}{8} \cdot \int_{0}^{L}\left(\frac{d \psi(s)}{d s}\right)^{4} \cdot d s \cdot w^{4}-\frac{E A}{2} \cdot \frac{u_{1}-u_{2}}{L} \cdot \int_{0}^{L}\left(\frac{d \psi(s)}{d s}\right)^{2} \cdot d s \cdot w^{2}
\end{gathered}
$$

The values of the horizontal displacements at the supports $\mathrm{u}_{1}$ and $\mathrm{u}_{2}$ can be found at the points where eqn. (10) is minimised:

$$
\begin{aligned}
& u_{1}=\frac{1}{2} \cdot k_{1 e q} \cdot \int_{0}^{L}\left(\frac{d \psi(s)}{d s}\right)^{2} \cdot \mathrm{ds} \cdot \mathrm{w}^{2} \quad \text { with } \quad k_{1 e q}=\frac{k_{L 2} \cdot k_{S}}{k_{L 1} \cdot k_{L 2}+k_{L 1} \cdot k_{S}+k_{L 2} \cdot k_{S}} \\
& u_{2}=\frac{1}{2} \cdot k_{2 e q} \cdot \int_{0}^{L}\left(\frac{d \Psi(s)}{d s}\right)^{2} \cdot \mathrm{ds} \cdot \mathrm{w}^{2} \quad \text { with } \quad k_{2 e q}=\frac{k_{L 1} \cdot k_{S}}{k_{L 1} \cdot k_{L 2}+k_{L 1} \cdot k_{S}+k_{L 2} \cdot k_{S}}
\end{aligned}
$$

where $\mathrm{k}_{\mathrm{L} 1}, \mathrm{k}_{\mathrm{L} 2}$ are the longitudinal stiffnesses of $\mathrm{K}_{\mathrm{L} 1}$ and $\mathrm{K}_{\mathrm{L} 2}$ and $\mathrm{k}_{\mathrm{S}}=\mathrm{EA} / \mathrm{L}$ is the axial beam stiffness.

From a further analytic decomposition of eqn. (10) and considering the results in eqns. (11) and (12), the strain energy absorbed by the left portion of the beam $\left(\mathrm{s}=0 \rightarrow \mathrm{s}_{\max }\right)$ can be expressed as:

$$
\begin{aligned}
& E_{\varepsilon, 0 \rightarrow \mathrm{s}_{\max }}=\left[L \cdot \mathrm{k}_{\mathrm{T}} \cdot \int_{0}^{s_{\max }}\left(\frac{d \psi(s)}{d s}\right)^{4} \mathrm{ds}+\frac{s_{\max }}{L} k_{T}\left(k_{1 e q}+k_{2 e q}\right)^{2}\left(\int_{0}^{L} \psi(s)^{2} \mathrm{ds}\right)^{2}-\right. \\
& \left.2 \cdot k_{T} \cdot\left(k_{1 e q}+k_{2 e q}\right) \cdot \int_{0}^{L} \psi(s)^{2} \cdot \mathrm{d} s \cdot \int_{0}^{s_{\max }} \psi(s)^{2} \cdot \mathrm{d} s\right] \cdot \frac{w^{4}}{8}
\end{aligned}
$$


From an energetical point of view, the left side of the beam behaves like a geometrical nonlinear spring, whose energy behaviour depends solely on $\mathrm{w}^{4}$ as in

$$
E_{\varepsilon, G N L}=\frac{k_{G N L}}{8} \cdot \frac{w^{4}}{s_{\max }^{2}}
$$

hence

$$
\begin{aligned}
& k_{G N L}=s_{\max }^{2}\left[L \cdot k_{T} \int_{0}^{s_{\max }}\left(\frac{d \psi(s)}{d s}\right)^{4} d s+\frac{s_{\max }}{L} k_{T}\left(k_{1 e q}+k_{2 e q}\right)^{2} .\right. \\
& \left.\left(\int_{0}^{L} \psi(s)^{2} d s\right)^{2}-2 k_{T}\left(k_{1 e q}+k_{2 e q}\right) \int_{0}^{L} \psi(s)^{2} d s \int_{0}^{s_{\max }} \psi(s)^{2} d s\right]
\end{aligned}
$$

After substituting the left side of the beam by a virtual spring $\mathrm{K}_{\mathrm{GNL}}$ with the same energy dissipating properties, the last step consists of defining the equivalent spring $\mathrm{K}_{\mathrm{H} 1}$, which groups both the connector and the beam spring in one element (see fig. 6).
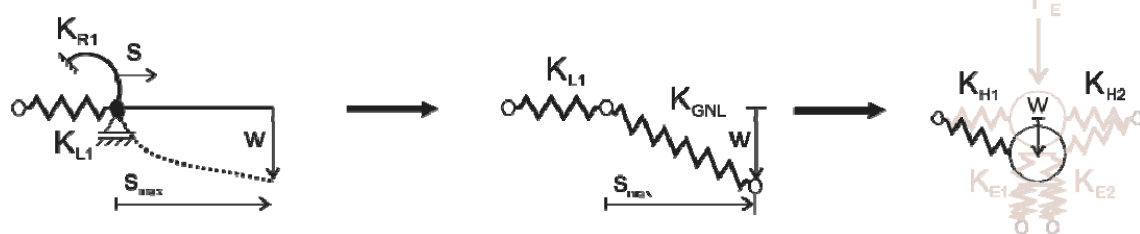

Figure 6: $\quad$ Reduction procedure in order to determine $\mathrm{K}_{\mathrm{H} 1}$; a) original system; b) connector + equivalent beam model; c) final model.

Without further demonstration, it can be shown that the spring $\mathrm{K}_{\mathrm{H} 1}$ is equivalent from an energetical point of view to the serial connection of both springs $\mathrm{K}_{\mathrm{L} 1}$ and $\mathrm{K}_{\mathrm{GNL}}$. Therefore the stiffness of the equivalent lateral spring $\mathrm{K}_{\mathrm{H} 1}$ can be calculated with the expression:

$$
k_{H 1}=\frac{1}{\frac{1}{k_{L 1}}+\frac{1}{k_{G N L}}}
$$

where $\quad \mathrm{k}_{\mathrm{L} 1} \quad$ is the lateral connector stiffness of spring $\mathrm{K}_{\mathrm{L} 1}$ and

$\mathrm{k}_{\mathrm{NGL}} \quad$ is the equivalent beam stiffness from eqn. (15)

In a very similar manner, the equivalent spring stiffness of $\mathrm{K}_{\mathrm{H} 2}$ can be derived to:

$$
k_{H 2}=\frac{1}{\frac{1}{k_{L 2}}+\frac{1}{k_{G N R}}}
$$

where

$$
\begin{aligned}
& k_{G N R}=\left(L-s_{\max }\right)^{2}\left[L \cdot k_{T} \int_{s_{\max }}^{L}\left(\frac{d \psi(s)}{d s}\right)^{4} d s+\frac{\left(L-s_{\max }\right)}{L} k_{T}\left(k_{1 e q}+k_{2 e q}\right)^{2} .\right. \\
& \left.\left(\int_{0}^{L} \psi(s)^{2} d s\right)^{2}-2 k_{T}\left(k_{1 e q}+k_{2 e q}\right) \int_{0}^{L} \psi(s)^{2} d s \int_{s_{\max }}^{L} \psi(s)^{2} d s\right]
\end{aligned}
$$




\subsection{Equivalent yielding criteria}

As exposed in section 2.2, dynamic system reduction concepts consider nonlinear system behaviour by the definition of a nonlinear force-deformation relation. With increasing accuracy demands and the desire for including arbitrary support conditions, this curve has become increasingly complicated, thus making its determination or computer implementation considerably complicated.

In the presented concept, the force-deformation relations of all four springs have been defined elastic-perfectly plastic, thus making its analysis and implementation very simple.

In vertical direction the yield criteria is defined according to Biggs' approach [3]. The yield load can be calculated by the principle of virtual work for each individual loading distribution. In case of a uniform linear load (most common assumption), the yield load $\mathrm{f}_{\mathrm{pl}}$ can be calculated with eqn. (19).

$$
f_{p l}=2 \cdot \frac{L \cdot M_{p l}+\left(L-s_{\max }\right) \cdot M_{p l, R 1}+s_{\max } \cdot M_{p l, R 2}}{\left(L-s_{\max }\right) \cdot s_{\max }}
$$

In the case of asymmetrical boundary conditions, the yield load can be distributed according to the weighting functions from Eqn. (9).

In membrane direction, the yield criteria for the springs $\mathrm{K}_{\mathrm{H} 1}$ and $\mathrm{K}_{\mathrm{H} 2}$ should be chosen as the minimum between the connector capacity and the beam axial resistance $\mathrm{N}_{\mathrm{pl}}$.

\section{Validation}

The behaviour of this new concept for an equivalent model reduction has been analysed with one example. Here the energy development of a strongly geometrical nonlinear system with high slenderness has been investigated under static load conditions. The results have been compared to an own implementation of the FEM with geometrically exact beam elements as described by Wriggers [10].

Given a beam with a length $\mathrm{L}$ of $10 \mathrm{~m}$ and a cross section of a U-60 with a steel grade of S235 for which the following cross-sectional properties apply: $\mathrm{EI}=51710 \mathrm{~N} \cdot \mathrm{m}^{2}, \mathrm{EA}=112.57 \mathrm{e} 6 \mathrm{~N}$, with respective yielding limits of $\mathrm{M}_{\mathrm{pl}}=296.89 \mathrm{e} 3 \mathrm{~N} \cdot \mathrm{m}$ and $\mathrm{N}_{\mathrm{pl}}=134.01 \mathrm{~N}$. An (unrealistic) high slenderness is taken here in order to appreciate the effects of membrane force activation before reaching any plastification limits. The member is uniformly loaded over the length with a load of $237.5 \mathrm{~N} / \mathrm{m}$, that corresponds to the plastic resistance of the beam assuming no catenary effects.

The boundary conditions have been set to free-free for the rotations and parametrically varied from free-free to fixed-fixed for the horizontal fixings.

In fig. 7 an analysis of the energy absorption dependency on the axial restrain is shown. Here three different models have been compared: (a) system modelled with linear beam elements, (b) system modelled with geometrically exact beam elements and (c) the equivalent system defined according to section 3 . 


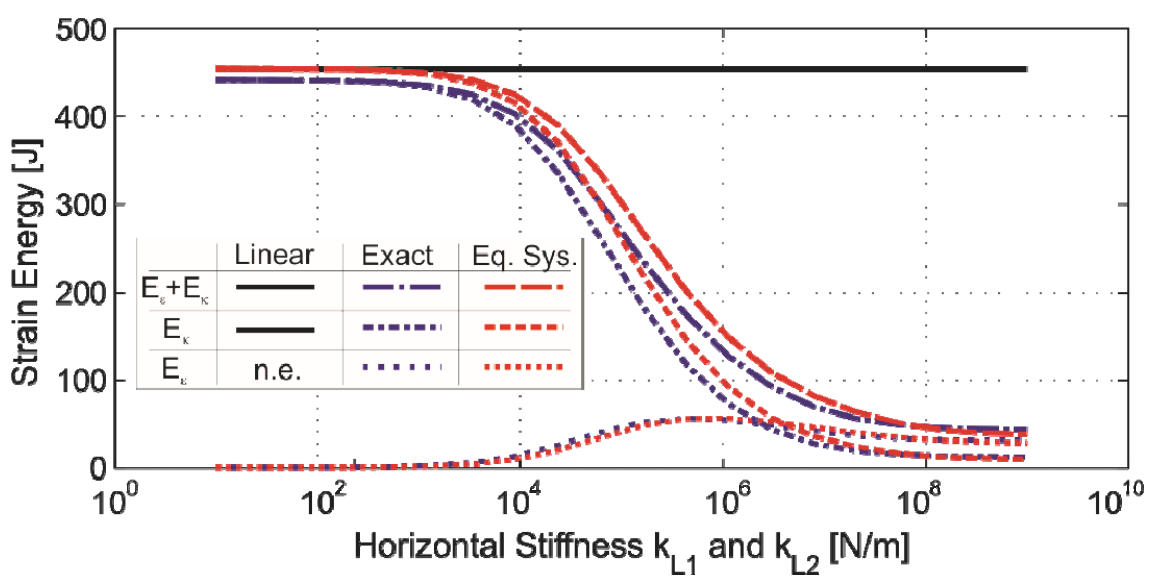

Figure 7: Comparison of the strain energy components with varying lateral connector stiffness.

The results show a very good agreement between the exact beam theory and the proposed equivalent model in terms of energy absorption capabilities.

\section{Conclusions}

In this paper a new simplified design method has been proposed based on a new concept for dynamic system reduction. The proposed methodology reduces an axially and rotationally arbitrary supported beam to a 2DOF system consisting of a mass and 4 springs as presented in fig. 5.

The new reduction concept exposed in section 3 is designed to explicitly address geometrical nonlinearities by means of two lateral springs with a nonlinear strain definition. Section 4 shows the very similar energetic behaviour of the reduced and the original system, even in the elastic domain, where most classical approaches do not consider any membrane action.

This approach opens new possibilities for the dynamic analysis of structures. As exposed in section 2, there is a large separation between complex FE calculations and the use of simplified models. The main disadvantage of classical reduction techniques is the fact that they cannot be integrated to more complex FE systems, so that the analysis remains for one isolated member. This limitation is mainly due to the fact that they would only offer one connection node to the rest of the FE system, thus not being able to separate membrane effects from bending effects or asymmetrical support action. The new approach overcomes this limitation by offering 4 connection nodes that separately consider the effects of bending and membrane action for both for the left and right support.

An implementation of an "equivalent beam" element in a FE system is possible and opens the possibility for parametric studies on larger structural models. 


\section{References}

[1] United States of America, Corps of Engineers, EM 1110-345-415; Design of structures to resist atomic weapons: Principles of Dynamic Analysis and Design, Washington D.C., 1957.

[2] C. H. Norris, R. J. Hansen, M. J. Holley, J. M. Biggs, S. Namyet and J. K. Minami, Structural Design for Dynamic Loads, New York: McGraw-Hill, 1959.

[3] J. M. Biggs, Introduction to structural dynamics, New York: McGrawHill, 1964.

[4] Det Norske veritas, DNV-RP-C204: Design against Accidental Loads, 2010.

[5] Norsok, N-004: Design of Steel Structures, Oslo, 2013.

[6] Fire and Blast Information Group (FABIG), Simplified methods for analysis of response to dynamic loading, Ascot: Steel Construction Institute, 2002.

[7] United States of America, Department of Defense, UFC 3-340-02: United Facilities Criteria: Structures to Resist the Effects of Accidental Explosions, 2008.

[8] W. Baker, P. Cox, J. Kulesz, R. Strehlow and P. Westine, Explosion Hazards and Evaluation, Oxford: Elsevier Science, 1983.

[9] M. Gündel, B. Hoffmeister and B. Hauke, Bemessung von Baustrukturen in Stahl- und Verbundbauweise für Anprall- und Explosionslasten, bauforumstahl e.V., Düsseldorf, 2010.

[10] Wriggers, Peter: Nonlinear Finite Element Methods. Berlin, Heidelberg: Springer Berlin Heidelberg, 2008. 